\title{
A GROUP-THEORETIC CHARACTERIZATION OF $M$-GROUPS
}

\author{
ALAN E. PARKS
}

\begin{abstract}
Groups having the property that all their complex irreducible characters are monomial are characterized in terms of the embedding of cyclic sections of the group.
\end{abstract}

Introduction. A character of a finite group $G$ is monomial if it is induced from a linear (degree-one) character of a subgroup of $G$. The group $G$ is an $M$-group if all of its complex irreducible characters (the set $\operatorname{Irr}(G))$ are monomial.

Isaacs [5, and 4, p. 67] and Berger [1, p. 43] have asked for a purely group-theoretic characterization of $M$-groups. We will now describe such a characterization; proofs. will be provided in $\S 1$.

If $M \triangleleft H \subseteq G$ with $H / M$ cyclic, we will say that $(H, M)$ is a pair. For $g \in G$ and $H \subseteq G$ we define $F_{H}(g)$ to be the set of commutators $\left[g, H \cap H^{g^{-1}}\right]$. We note that $F_{H}(g) \subseteq H$ : indeed if $h \in H \cap H^{g^{-1}}$, then $h=g k g^{-1}$ for some $k \in H$. Then $[g, h]$ $=g^{-1} h^{-1} g h=k^{-1} h \in H$. If $(H, M)$ is a pair, we will say that it is a good pair in $G$, if $F_{H}(g) \nsubseteq M$ for all $g \in G-H$.

If $(H, M)$ and $(K, L)$ are good pairs, we will say they are related in $G$ if there is $g \in G$ such that $H^{g} \cap L=K \cap M^{g}$. Let $S_{G}$ be the equivalence relation on good pairs in $G$ generated by the relation of being related. Let $m_{G}$ be the number of distinct classes of $S_{G}$.

We identify a relation on the elements of $G$. We say $x \sim y$ for $x, y \in G$ provided the two cyclic groups $\langle x\rangle$ and $\langle y\rangle$ are conjugate in $G$. Clearly $\sim$ is an equivalence relation. (The equivalence classes of $\sim$ are sometimes called the rational conjugacy classes of $G$.) Let $n_{G}$ be the number of $\sim$ equivalence classes.

THEOREM. We have $m_{G} \leqslant n_{G}$ with equality if and only if $G$ is an M-group.

The Theorem is the promised characterization.

We would like to thank T. R. Berger for pointing out an error in an earlier version of this paper.

1. Proofs. Let $J$ and $L$ be subgroups of a group $G$. A set of representatives $T$ for the double cosets of $J$ and $L$ in $G$ will be called a $J, L$ transversal in $G$.

For a character $\theta$ of $J$ and $x \in G$ we define a character $\theta^{x}$ of $J^{x}$ by the formula

$$
\theta^{x}(g)=\theta\left(x g x^{-1}\right) \quad \text { for } g \in J^{x} .
$$

The following appears as V.16.9 of [3].

Received by the editors February 16, 1984 and, in revised form, August 25, 1984.

1980 Mathematics Subject Classification. Primary 20B10. 
1.0 TheOREM (MACKey). Let $J, L \subseteq G$. Let $T$ be a $J, L$ transversal in $G$. Let $\theta$ and $\varphi$ be characters of $J$ and $L$, respectively. Then

$$
\left[\theta^{G}, \varphi^{G}\right]=\sum_{g \in T}\left[\left(\theta^{g}\right)_{J^{g} \cap L}, \varphi_{J^{g} \cap L}\right]
$$

For any pair $(H, M)$, there is a linear $\lambda \in \operatorname{Irr}(H)$ with $M$ equal to the kernel of any representation affording $\lambda$ (we write $M=\operatorname{ker}(\lambda)$ ). We will say that $\lambda$ proceeds from $(H, M)$.

1.1 Proposition. Let $(H, M)$ be a pair with $H \subseteq G$. Let $\lambda$ proceed from $(H, M)$. Then $(H, M)$ is a good pair in $G$ if and only if the induced character $\lambda^{G}$ is irreducible.

Proof. Let $\lambda$ proceed from $(H, M)$.

Claim. If $x \in G$ then $\left[\left(\lambda^{x}\right)_{H^{x} \cap H}, \lambda_{H^{x} \cap H}\right]=1$ if and only if $F_{H}\left(x^{-1}\right) \subseteq M$.

Proof. Put $K=H^{x} \cap H$. Then $\lambda_{K}$ and $\left(\lambda^{x}\right)_{K}$ are linear characters of $K$. Hence $\left[\left(\lambda^{x}\right)_{K}, \lambda_{K}\right]=1$ if and only if $\left(\lambda^{x}\right)_{K}=\lambda_{K}$.

Let $g \in K$ and suppose $\left(\lambda^{x}\right)_{K}=\lambda_{K}$. Then $\lambda^{x}(g)=\lambda(g)$, so then $\lambda\left(x g x^{-1}\right)=\lambda(g)$. Since $\lambda$ is linear, this proves that $\lambda\left(x g x^{-1} g^{-1}\right)=1$, and so $\left[x^{-1}, g^{-1}\right] \in \operatorname{ker}(\lambda)=M$. Thus $F_{H}\left(x^{-1}\right)=\left[x^{-1}, K\right] \subseteq M$. Conversely, if $F_{H}\left(x^{-1}\right) \subseteq M$, then $\lambda\left(x g x^{-1}\right)=\lambda(g)$ for all $g \in K$. Then $\left(\lambda^{x}\right)_{K}=\lambda_{K}$, as needed.

Now $\lambda^{G} \in \operatorname{Irr}(G)$ iff $\left[\lambda^{G}, \lambda^{G}\right]=1$. Let $\lambda^{G}$ be irreducible and choose $x \in G-H$. Then there is an $H, H$ transversal $T$ in $G$ with $1, x \in T$. By Theorem 1.0

$$
\left[\lambda^{G}, \lambda^{G}\right] \geqslant\left[\lambda_{H}, \lambda_{H}\right]+\left[\left(\lambda^{x}\right)_{H^{x} \cap H}, \lambda_{H^{x} \cap H}\right] .
$$

So then $\left[\left(\lambda^{x}\right)_{H^{x} \cap H}, \lambda_{H^{x} \cap H}\right]=0$. By the Claim, $F_{H}(x) \nsubseteq M$. This proves one direction of Proposition 1.1.

Suppose for all $x \in G-H$ that $F_{H}(x) \nsubseteq M$. By the Claim, $\left[\left(\lambda^{x}\right)_{H^{x} \cap H}, \lambda_{H^{x} \cap H}\right]$ $=0$ for all $x \in G-H$. Then using Theorem 1.0 we see that $\left[\lambda^{G}, \lambda^{G}\right]=\left[\lambda_{H}, \lambda_{H}\right]=1$. This completes the proof of Proposition 1.1.

1.2 Proposition. If $(H, M)$ and $(K, L)$ are good pairs, then they are related if and only if there are characters $\lambda$ and $\mu$ proceeding from $(H, M)$ and $(K, L)$, respectively, such that $\lambda^{G}=\mu^{G}$.

Proof. Assume $\lambda$ and $\mu$ proceed from the good pairs $(H, M)$ and $(K, L)$, respectively, and suppose that $\lambda^{G}=\mu^{G}$.

Let $T$ be an $H, K$ transversal in $G$. By Theorem 1.0 , since $\left[\lambda^{G}, \mu^{G}\right] \neq 0$, we have

$$
\left[\lambda_{H^{x} \cap K}^{x}, \mu_{H^{x} \cap K}\right] \neq 0 \text { for some } x \in T \text {. }
$$

Now $\left(\lambda^{x}\right)_{H^{x} \cap K}$ and $\mu_{H^{x} \cap K}$ are linear and we conclude that $\left(\lambda^{x}\right)_{H^{x} \cap K}=\mu_{H^{x} \cap K}$. In particular, their kernels are the same, that is

$$
M^{x} \cap H^{x} \cap K=L \cap H^{x} \cap K .
$$

This is clearly $M^{x} \cap K=L \cap H^{x}$. Hence $(H, M)$ and $(K, L)$ are related.

Conversely, assume $H^{x} \cap L=K \cap M^{x}$ for some $x \in G$. Then $L \cap H^{x} \cap K=$ $H^{x} \cap K \cap M^{x}$; call this group $N$. Now $\left(H^{x} \cap K\right) / N$ is isomorphic to a subgroup of 
$K / L$ which is cyclic. Thus there is a faithful linear $\nu \in \operatorname{Irr}\left(\left(H^{x} \cap K\right) / N\right)$. Since $N=\left(H^{x} \cap K\right) \cap L, \nu$ extends to $\mu \in \operatorname{Irr}(K)$ with $L=\operatorname{ker}(\mu)$, and since $N=\left(H^{x}\right.$ $\cap K) \cap M^{x}, \nu$ extends to $\lambda^{x} \in \operatorname{Irr}\left(H^{x}\right)$, where $\lambda \in \operatorname{Irr}(H)$ and $M=\operatorname{ker}(\lambda)$.

Including $x$ in an $H, K$ transversal in $G$, Theorem 1.0. shows that

$$
\left[\lambda^{G}, \mu^{G}\right] \geqslant\left[\left(\lambda^{x}\right)_{H^{x} \cap K}, \mu_{H^{x} \cap K}\right]=[\nu, \nu]=1 .
$$

Because $(H, M)$ and $(K, L)$ are good pairs, $\lambda^{G}$ and $\mu^{G}$ are irreducible. Thus $\lambda^{G}=\mu^{G}$ as needed.

We remark that Proposition 1.2 shows that being related is actually an equivalence relation on the set of good pairs, and so the equivalence classes of $S_{G}$ are precisely the classes of related good pairs. It might be interesting to find a purely group-theoretic proof that being related is an equivalence relation.

The proof of the Theorem is close at hand. We say $\chi, \psi \in \operatorname{Irr}(G)$ are Galois conjugate if there is $\sigma \in \operatorname{Aut}(\mathbf{C})$ such that $\chi^{\sigma}=\psi$. If $s(\chi)$ is the Schur index of $\chi$ over the rationals (see [4, Chapter 10]), then $s(\chi)$ times the $\operatorname{sum} \operatorname{sp}(\chi)$ of the distinct Galois conjugates of $\chi$ in $\operatorname{Irr}(G)$ is the character afforded by an irreducible, rational representation of $G$. By [4, Theorem 9.21], all irreducible, rationally-afforded characters of $G$ arise as $s(\chi) \operatorname{sp}(\chi)$ for $\chi \in \operatorname{Irr}(G)$. By the Berman-Witt Theorem [2, 42.9], the number $n_{G}$ defined in the Introduction is the same as the number of distinct, irreducible, rationally-afforded characters of $G$, and thus $n_{G}$ is the number of Galois conjugacy classes of $\operatorname{Irr}(G)$.

Proof of THEOREM. By the preceding discussion, it suffices to show that there is a one-to-one correspondence between the set of Galois conjugacy classes of monomial elements of $\operatorname{Irr}(G)$ and classes of related good pairs.

Let $\left(H_{i}, M_{i}\right), 1 \leqslant i \leqslant m_{G}$, be a set of representatives of the clases of related good pairs in $G$. For each $i$, let $\lambda_{i}$ proceed from $\left(H_{i}, M_{i}\right)$; then $\lambda_{i}^{G} \in \operatorname{Irr}(G)$ by Proposition 1.1. To complete the proof, we will show that, given monomial $\chi \in \operatorname{Irr}(G)$, there is a unique $i$ for which $\chi$ is Galois conjugate to $\lambda_{i}^{G}$.

Indeed, suppose $\chi=\mu^{G}$ where $\mu \in \operatorname{Irr}(H), H \subseteq G$, and $\mu(1)=1$. Put $M=\operatorname{ker}(\mu)$; then by Proposition 1.1, $(H, M)$ is a good pair from which $\mu$ proceeds. Now $(H, M)$ is related to some $\left(H_{i}, M_{i}\right)$ and Proposition 1.2 grants $\mu^{\prime}$ proceeding from $(H, M)$ and $\lambda^{\prime}$ proceeding from $\left(H_{i}, M_{i}\right)$ with

$$
\left(\mu^{\prime}\right)^{G}=\left(\lambda^{\prime}\right)^{G} .
$$

The characters $\mu^{\prime}$ and $\mu$ faithfully represent the same cyclic group. By the irreducibility of the cyclotomic polynomials there is $\sigma \in \operatorname{Aut}(\mathbf{C})$ such that $\mu^{\sigma}=\mu^{\prime}$. Similarly there is $\tau \in \operatorname{Aut}(\mathbf{C})$ with $\left(\lambda^{\prime}\right)^{\tau}=\lambda_{i}$. Compute

$$
\begin{aligned}
\chi^{\sigma \tau} & =\left(\left(\mu^{\sigma}\right)^{G}\right)^{\tau}=\left(\left(\mu^{\prime}\right)^{G}\right)^{\tau} \\
& =\left(\left(\lambda^{\prime}\right)^{G}\right)^{\tau} \quad \text { using }(*) \\
& =\left(\left(\lambda^{\prime}\right)^{\tau}\right)^{G}=\lambda_{i}^{G} .
\end{aligned}
$$

Thus $\chi$ is Galois conjugate to $\lambda_{i}^{G}$. 
As for the uniqueness of $i$, if $\chi$ is also conjugate to $\lambda_{j}^{G}$, then there is $\sigma \in \operatorname{Aut}(\mathbf{C})$ with $\left(\lambda_{i}^{G}\right)^{\sigma}=\lambda_{j}^{G}$. Thus $\left(\lambda_{i}^{\sigma}\right)^{G}=\lambda_{j}^{G}$. Observe that $\lambda_{i}^{\sigma}$ proceeds from $\left(H_{i}, M_{i}\right)$, and then Proposition 1.2 allows us to conclude that $\left(H_{i}, M_{i}\right)$ and $\left(H_{j}, M_{j}\right)$ are related. This forces that $i=j$. The proof is complete.

\section{REFERENCES}

1. T. Berger, Proc. Sympos. Pure Math., vol. 37, Amer. Math. Soc., Providence, R. I. 1980.

2. C. W. Curtis and I. Reiner, Representation theory of finite groups and associative algebras, Interscience, New York, 1962.

3. B. Huppert, Endliche Gruppen. I, Springer-Verlag, Berlin, 1967.

4. I. M. Isaacs, Character theory of finite groups, Academic Press, New York, 1976.

5 . , Primitive characters, normal subgroups, and M-groups, Math. Z. 177 (1981), 267-284.

Department of Mathematics, Michigan State University, East Lansing, Michigan 48824

Current address: Department of Mathematics, Ohio University, Athens, Ohio 45701 